\title{
An In Vitro Assessment of the Cytotoxic and Apoptotic Potency of Silymarin and Silymarin Loaded Solid Lipid Nanoparticles on Lung and Breast Cancer Cells
}

\author{
Canan Vejselova Sezer* \\ Department of Biology, Faculty of Science, Eskisehir Technical University, 26470 \\ Eskisehir, Turkey
}

\begin{abstract}
A B S T R A C T
Cancer disease is a major cause of death worldwide in the last few decades. Recently, the risk of cancer and its mortality rates have increased both in economically less and highly developed countries. Two of the leading causes of the death of cancer are lung and breast cancers among males and females, respectively. A variety of drugs are in use for cancer treatment but their recruiting properties are limited because of the developing resistance and/or finiteness of bioavailability. Current research is aimed at synthesis of a new effective agent of a different type, silymarin-loaded solid lipid nanoparticles and investigation of its effectiveness on treatment of lung and breast cancers. In this respect, these nanoparticles and silymarin were elucidated by using MTT assay for cytotoxicity, annexin-V analyses for cell death mechanism and confocal and transmission electron microscopies for morphological and ultrastructural changes. Based upon our findings, it can be concluded that silymarin-loaded solid lipid nanoparticles significantly reduced the growth of A549 and MCF-7 cells compared to silymarin. Also these nanoparticles induced apoptosis both in A549 and MCF-7 cells in higher percentages than that of silymarin. In microscopic investigations, it was shown clearly that apoptotic cell death hallmarks in silymarin and silymarin-loaded solid lipid nanoparticles treated cells, being more prevalent for the latter. These findings suggested improved bioavailability of silymarin in nanoparticle formulation, consequently silymarin-loaded solid lipid nanoparticles could be considered as a useful drug delivery system for silymarin that has poor watersolubility and an excellent candidate for anticancer drug development.
\end{abstract}

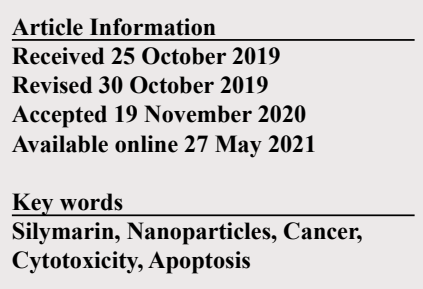

ation

Silymarin, Nanoparticles, Cancer,

Cytotoxicity, Apoptosis

\section{INTRODUCTION}

$\mathrm{L}$ ung cancer is the leading cause of death among males in developed and less developed countries. The disease with maximal mortality among females in less developed countries is reported as breast cancer (Torre et al., 2015). Classical chemotherapy alone became ineffective in cancer treatment as to developing resistance by many of cancer cell types (Liu, 2004; Obradovic et al., 2013). Thus, novel agents offering theranostic potency are required. Eugenol, eucalyptol and camphor promising anticancer potency as natural agents were elucidated in parental and drugresistant human lung cancer cell lines (Özkan and Erdoğan, 2013). It was proved that these agents are effective in apoptosis promotion but more effective ones are needed to be found (Özkan and Erdoğan, 2013). Apoptosis is defined as a programmed cell death that occurs via cell-derived or extracellular initiators that mainly regulates the tissue

\footnotetext{
* Corresponding author: cananveyselova@gmail.com 0030-9923/2021/0004-1407 \$9.00/0

Copyright 2021 Zoological Society of Pakistan
}

homeostasis of the body. Triggering apoptotic cell death is one of the main research focuses of drug development with anticancer activities. In this respect, research on finding the mode of cell death starts with detection of the physiological and morphological changes that refer to apoptosis such as membrane disintegration, chromatin and nuclear condensation of cells exposed to an anticancer agent (Kerr et al., 1972).

Silymarin aflavonolignan that is a bioactive constituent of Silybum marianum possesses chemosensitizing, antioxidant and anti-inflammatory potentials. In addition, as a phytochemical, it has concentration-related antiapoptotic and cell transporters altering, free radical scavenging, lipid peroxidation inhibitory and cellular membrane stability enhancing and steroid-like activities (Karimi et al., 2011; Prasad et al., 2016; Montgomery et al., 2016). Recently, compounds of natural sources have been reported to inhibit hyperproliferation of the cells, their neoplastic transformation as well as carcinogenesis (Agarwal et al., 2006). Phytochemicals as compounds of natural resources and their usefulness for cancer prevention and/or treatment are on the main focus of cancer research 
(Aljuffali et al., 2016). The antioxidant, anti-inflammatory and proapoptotic properties are implying their anticancer efficacy (Iriti and Faoro, 2009). Food like fruits and vegetables has different kind of phytochemicals that have anticancer potency (Montgomery et al., 2016). Currently, silymarin is used as a hepatoprotective drug supplement in both clinical and experimental models due to its above mentioned properties (Karimi et al., 2011; Eminzade et al., 2008). Silymarin has been declared as safe/nontoxic/with low toxicity in human studies (Eminzade et al., 2008). However, the molecular mechanisms underlying the bioactivity of silymarin and its drug/food interactions have still remained unclear (Mayer et al., 2005). In addition, anticancer activities of the compound are limitedly investigated for some cancer types in vivo and in vitro. In the studies carried out by Agarwal et al. (2006) and Malewicz et al. (2006) the chemo-preventive efficiency of silymarin on breast cancer was elucidated but they considered silymarin as conflicting in mammary carcinogenesis (Agarwal et al., 2006; Malewicz et al., 2006). To unravel the deeper mechanism of the anticancer activities of silymarin and its derivatives in mammary and other type of cancers, deeper investigations are needed to be performed. In the last few decades, investigations on cancer treatment by using natural agents with low toxicity and side effects are in rise. Silymarin has many bioactive properties as to balance the survival and death/apoptosis as well as to be anti-inflammatory, anti-metastatic under the cells. The cytoprotective, cancer protective and anticancer efficacies of silymarin have been investigated under breast, prostate, cervical, liver, colon and lung cancers (Gezgin et al., 2013). Despite the usefulness of silymarin in many cancer research, it has limitations on the bioavailability due to its quite low solubility in water (Provinciali et al., 2007). To diminish this limitation, novel approaches on preparing sophisticated formulations of silymarin for cancer therapy are required. Recent approaches on enhancement of the drug bioavailability have been redirected on preparing nanoscale formulations. Their sizes vary from $1 \mathrm{~nm}$ to several hundred nanometers and can be used as carriers to conquer the limitations of drugs (Jabir et al., 2012). Nanoparticles as drug carriers are considered to provide controlled, targeted delivery and high bioavailability of drugs (Almeida et al., 2015). As one of that kind of carriers, solid lipid nanoparticles (SLNs) were involved to eliminate the limitations and lower the side effects of natural products that were used as drugs and their derivatives (Seyfoddin et al., 2015). When used orally, SLNs loaded with drugs in their monolayer phospholipid shell, are beneficial as to their enhanced theranostic opportunity and pharmacokinetics such as lengthening half-life, stability, reduced side effects, clearance, mean residence time and enhanced bioavailability etc. (Jabir et al., 2012; Li and Huang, 2008; Geszke-Moritz and Moritz, 2016; Chih-Hung et al., 2017). Furthermore, they lower the risk of toxicity with their biodegradable lipid content and offer safe and efficient drug delivery especially for drugs with limited solubility in water (Jaafar-Maalej et al., 2011). SLNs are preferred among other colloid drug carrier systems due to their benefit for being low-priced and easiness for production in big commercial amounts (Harde et al., 2011).

In this study, it is aimed to prepare solid lipid nanoparticles carrying commercial silymarin by using hot homogenization method and to examine their cytotoxicity and apoptosis promoting capability in comparison with silymarin alone on human lung adenocarcinoma and human breast cancer cells.

\section{MATERIALS AND METHODS}

\section{Materials}

MCF-7 (ATCC ${ }^{\circledR}$ HTB-22 ${ }^{\mathrm{TM}}$ ) and A549 (ATCC ${ }^{\circledR}$ CCL-185 ${ }^{\mathrm{TM}}$ ) purchased from the American Type Culture Collection (Manassas, USA). The silymarin powder (S0292), MTT (3-(4,5-dimethylthiazol-2-yl)-2,5 diphenyl2H-tetrazolium bromide) (M2003), fetal bovine serum, penicillin-streptomycin, dimethyl sulfoxide (DMSO) were from Sigma-Aldrich (St. Louis, USA), and Roswell Park Memorial Institute medium (RPMI-1640) was obtained from GIBCO (Grand Island, USA). Compritol and polyoxyethylene sorbitan monooleate (Tween 80 ) were purchased from Merck Schuchardt (Darmstadt, Germany).

Synthesis of silymarin-loaded solid lipid nanoparticles (SLNS)

Hot homogenization method described before by Muller et al. (2000) (www.sigmaaldrich.com, accessed 24 March 2018) was used for the preparation of silymarinloaded SLNs. Briefly, used lipid agent was kept in a hot water bath in a glass well until it melted $\left(80^{\circ} \mathrm{C}\right)$. Silymarin $(5 \%)$ was added in the melted lipid and tween $80(3 \%)$ was included as surfactant. $87 \%(\mathrm{w} / \mathrm{w})$ of the suspension consists of bidistilled water. This mixture was homogenized via using Ultra-Turrax at $20500 \mathrm{rpm}$ (T25, Janke and Kunkel IKA ${ }^{\circledR}$, Germany). After it cooled down at room temperature and the filtration was performed, the prepared suspension was stored at $+4{ }^{\circ} \mathrm{C}$ in dark drug bottles.

\section{Analyses of characteristics of the particles}

Particle size, polydispersity index, zeta potential and electrical conductivity of the silymarin-loaded SLNs and silymarin were measured in distilled water in triplicate 
by using green zeta cells at $25^{\circ} \mathrm{C}$ under Malvern Zetasizer Nano ZS (Malvern Instruments). For polydispersity index measurement, the Nano Zetasizer (ZS, Malvern, UK) was adjusted to a fixed angle of $90^{\circ}$ and $25^{\circ} \mathrm{C}$ temperature. To measure the potential of the samples, bidistilled water was used with electrical conductivity of $50 \mu \mathrm{S} / \mathrm{cm}$ (adjusted with $\mathrm{NaCl}$ ).

Scanning electron microscopy (SEM) for visualization of the morphological characteristics of the particles

The shape of the particles was imaged by SAM. For preparation of SAM samples, suspensions of silymarinloaded SLNs and silymarin were dropped onto the carbon stickers on the separate sample holders. Samples were allowed to air for drying. Prepared samples were placed in argon atmosphere and coated with gold for SEM imaging.

\section{Cell culture}

Breast MCF-7 (ATCC® HTB-22 ${ }^{\mathrm{TM}}$ ) cancer cells and lung A549 (ATCC ${ }^{\circledR}$ CCL-185 ${ }^{\mathrm{TM}}$ ) cancer cells were cultured in phenol red free RPMI 1640 medium (Gibco, USA) containing penicillin-streptomycin (1\%) (Gibco, USA), fetal bovine serum (10\%, Lonza, Belgium) at $37^{\circ} \mathrm{C}$ in a humidified atmosphere supplemented with $\mathrm{CO}_{2} 5 \%$ ). Cells with a confluency of $80 \%$ were used in all of the investigations.

\section{Cytotoxicity assay}

Commercial silymarin used in this study was containing $(45 \% \mathrm{w} / \mathrm{w})$ silybin (A and B) isosilybin (A and B), silychristin, silydianin taxifolin finally apigenin 7-glucoside (Muller et al., 2000). The effect of silymarin on the viability of breast and lung cancer cells was determined by 3-(4,5-dimethylthiazol-2-yl)-2,5diphenyltetrazolium bromide (MTT) assay. In this respect, a stock solution of silymarin was prepared (in DMSO with 1\% final concentration) (Polyak et al., 2010; Gharagozloo et al., 2013). The prepared silymarin-loaded SLNs were suspended in fresh culture medium. These solutions were applied on different concentrations $(2,34-150 \mu \mathrm{M})$ on A549 and MCF-7 cells plated into 96-well plates $\left(1 \times 10^{5}\right.$ cells per well). Cells were incubated with the agents for 24 $\mathrm{h}$ at $37^{\circ} \mathrm{C}$ in a humidified atmosphere of $5 \% \mathrm{CO}_{2}$ in air. At the end of incubation period, $20 \mu \mathrm{L}$ of MTT solution $(5 \mathrm{mg} /$ $\mathrm{mL}$ ) was added to each well and incubated for $2 \mathrm{~h}$ under the same conditions. After the incubation, the medium from each well was aspired and $200 \mu \mathrm{L}$ of dimethyl sulphoxide was added to the wells, mixed thoroughly and kept at room temperature for 10 minutes. Plates were read on an ELISA reader at a wavelength of $570 \mathrm{~nm}(\mathrm{n}=3)$.

\section{Confocal imaging for apoptosis visualization}

In order to examine the nuclear morphology and membrane integrity of cells, two fluorescent dyes, phalloidin and acridine orange were used respectively. MCF-7 and A549 cells were seeded in 6-well culture plates at a density of $3 \times 10^{5}$ per well and treated with $\mathrm{IC}_{50}$ inhibition concentrations of silymarin and silymarin loaded solid lipid nanoparticles at $37^{\circ} \mathrm{C}$ for $24 \mathrm{~h}$.

At the end of the treatment period, cells were incubated with phalloidin and acridine orange at room temperature for $20 \mathrm{~min}$ in the dark. Stained cell was mounted to slides and imaged with a confocal microscope.

\section{Transmission electron microscopy (TEM)}

MCF-7 and A549 cells treated $\mathrm{IC}_{50}$ value of silymarin and silymarin-loaded solid lipid nanoparticles for $24 \mathrm{~h}$ were fixed in glutaraldehyde $(2.5 \%$, in $0.1 \mathrm{M}$ phosphate buffer ( $\mathrm{pH}$ 7.4) and post fixed in osmium tetroxide $(2 \%)$. After the fixation, cell samples were dehydrated in graded ethyl alcohol $(70 \%, 90 \%, 96 \%$, and absolute alcohol) then embedded in Epon 812 epoxy and sectioned on ultramicrotome. Thin sections were prepared using a glass knife to a maximum thickness of $100 \mathrm{~nm}$. Thin sections were stained in lead citrate and uranyl acetate and evaluated under TEM.

\section{Annexin-V analysis}

Mode of cell death triggered by silymarin and silymarin-loaded SLNs was elucidated by annexin-V staining in A549 and MCF-7 cells. In this manner, all test cell groups (untreated, silymarin and silymarinloaded SLNs treated cells prepared in triplicate) were harvested by using trypsin and $100 \mu \mathrm{L}$ of each group were transferred to separate eppendorf tubes. These cell groups were incubated with $100 \mu \mathrm{L}$ of annexin-V reagent/sample for $20 \mathrm{~min}$ at room temperature in dark as described in the user manual of Muse ${ }^{\circledR}$ Annexin-V and Dead Cell Assay Kit and analyzed by using Muse ${ }^{\mathrm{TM}}$ Cell Analyzer (Merck, Millipore, Hayward, California, USA).

\section{Statistical evaluations}

For statistical analysis of our results, one-way ANOVA was performed for multiple comparisons Tukey post-test of Graphpad Prism 6.0 for Windows and the data was expressed as means \pm SDs. $p<0.05$ and $p<0.01$ values were considered as statistically significant.

\section{RESULTS AND DISSCUSSION}

Cancer therapy by using the classical chemotherapeutics has too many side effects also exhibit high level of toxicity for patients. Novel approaches on cancer therapy imply the use of phytochemicals and/ or other nanoscale sized natural products that offer low 
toxicity and fewer side effects. These nano formulations are more effective in low doses than the normal sized agents in cancer treatment (Numanoğlu and Tarımc1, 2006). In this study, silymarin-loaded SLNs were prepared and characterized by measuring the particle size, zeta potential, polydispersity index and conductivity. In addition, the shape of nanoparticles was visualized by using scanning electron microscopy method. The size of the particles refers to the physical stability and activity of SLNs (Numanoğlu and Tarımc1, 2006). The size of the silymarin-loaded SLNs was measured $92.5 \mathrm{~nm}$ that is about ten times smaller compared to the size of silymarin particles $(997.2 \mathrm{~nm})$ that implies to a nano formulationderived to reduce of the size of silymarin particles (Table I). Zeta potential was measured to examine the storage stability of SLNs. Zeta potential values of silymarinloaded SLNs were presented in Table 3. As it can be seen at Table II, silymarin-loaded SLNs were negatively charged and had a potential about $-11.7 \mathrm{mV}$. This value refers to a relatively good dispersion quality and physical stability (Venkateswarlu and Manjunath, 2004). This potential may be affected by storage conditions and aggregation may occur. Aggregation in charged particles does not rarely occur due to the electric repulsion, though (Al-Haj and Rasedee, 2009). Other important values of stability for SLNs dispersion are electrical conductivity and polydispersity index (Al-Haj and Rasedee, 2009). These values were determined in the safe level for a relatively good stability of particles (Tables I and II). Moreover, scanning electron microscopic evaluation results indicated the spherical particles of SLNs loaded with silymarin while a cubic-like shape for silymarin particles (Fig. 1).

The short time treatment with nanoparticles led to the inhibition of the growth of A549 and MCF-7 cells. Growth inhibition of A549 cells exposed to different silymarin concentrations raised when applied concentration increased during $24 \mathrm{~h}$ of incubation. $\mathrm{IC}_{50}$ value for these cells was 33 $\mu \mathrm{M}$ (Fig. 2). Also, viability of treated A549 cells decreased in accordance with the dose and $\mathrm{IC}_{50}$ value was calculated as $25 \mu \mathrm{M}$ for $24 \mathrm{~h}$ of silymarin-loaded SLNs. The half maximal inhibitory concentration $\left(\mathrm{IC}_{50}\right)$ of silymarin under MCF-7 cells for $24 \mathrm{~h}$ was not found at the range of applied concentrations (Fig. 3). This might be implying the limited bioavailability of silymarin. However, $\mathrm{IC}_{50}$ concentration of SLNs for MCF-7 cells $(18 \mu \mathrm{M})$ was found to be lower than that of SLNs for A549 cells. This might be attributed to the difference between the used cell lines and their sensitivity to applied nanoparticles that are needed to be figured out with further investigations. Similar to our results, Deep et al. (2007) have considered that silymarin and its nano formulation have been antiproliferative in human prostate carcinoma LNCap and 22Rv1 cell lines.
Silymarin loaded SLNs showed an antigrowth efficacy on A549 and MCF-7 cells with half-maximal inhibitory concentrations ranging at $18-25 \mu \mathrm{M}$, respectively. Our results refer to a good anticancer potency in lower doses for silymarin-loaded SLNs for A549 and MCF-7 cells.

Table I. The particle size and polydispersity index of silymarin and silymarin loaded solid lipid nanoparticles.

\begin{tabular}{|c|c|c|c|c|}
\hline \multirow{2}{*}{$\begin{array}{l}\text { Formula- } \\
\text { tions }\end{array}$} & \multicolumn{2}{|c|}{ Particle size (nm) } & \multicolumn{2}{|c|}{ Polydispersity index (PI) } \\
\hline & $\begin{array}{l}\text { Medium } \\
(\mathrm{n}=3)\end{array}$ & S.D. & $\begin{array}{l}\text { Medium } \\
(\mathrm{n}=3)\end{array}$ & S.D. \\
\hline Silymarin & 997.2 & 53.73 & 0.752 & 0.0001 \\
\hline $\begin{array}{l}\text { Silymarin } \\
\text { loaded SLN }\end{array}$ & 92.54 & 1.03 & 1.000 & 1.063 \\
\hline
\end{tabular}
$3)$.

Table II. The zeta potential and electrical conductivity of silymarin and silymarin-loaded solid lipid nanoparticles.

\begin{tabular}{lllll}
\hline Formulations & \multicolumn{2}{l}{$\begin{array}{l}\text { Zeta potential } \\
(\mathbf{m V})\end{array}$} & $\begin{array}{l}\text { Electrical conductivi- } \\
\mathbf{t y}\left(\mathbf{m S} \cdot \mathbf{c m}^{-\mathbf{1}}\right)\end{array}$ \\
\cline { 2 - 5 } & $\begin{array}{l}\text { Medium } \\
(\mathbf{n = 3})\end{array}$ & S.D. & $\begin{array}{l}\text { Medium } \\
(\mathbf{n}=\mathbf{3})\end{array}$ & S.D. \\
\hline Silymarin & -19.2 & 6.59 & 0.01 & 0.0005 \\
Silymarin loaded & -11.7 & 1.09 & 0.01 & 0.002 \\
SLN & & & & \\
Data is shown as mean values and standard deviation $(\mathrm{n}=3)$.
\end{tabular}
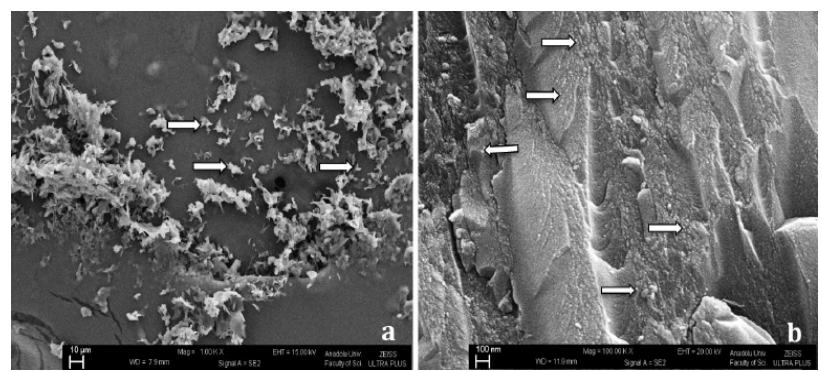

Fig. 1. The shape and morphology of silymarin (a, arrowssilymarin particles) and silymarin loaded SLN (b, arrowssilymarin-loaded SLN particles).

On the confocal images of A549 cells exposed to $\mathrm{IC}_{50}$ value of silymarin alone for $24 \mathrm{~h}$, holes on cytoskeleton, nuclei with condensed chromatin were detected as morphological changes. On A549 cells treated with silymarin-loaded SLNs were seen morphological alterations as fragmented cytoskeleton, pyknotic nucleus, chromatin condensation as well as holes on cytoskeleton 
(Fig. 4). Similarly, in MCF-7 cells exposed to silymarin alone, condensed chromatin, fragmented nuclei and diminished number of cells in a typical cell cluster were found. Moreover, in silymarin-loaded SLN treated MCF-7 cells were detected to be shrunken and excessive chromatin condensation and highly perforated cytoskeleton were seen alterations in these cells (Fig. 5). Our confocal finding implies apoptosis in both cell lines treated with silymarin alone and silymarin loaded SLNs, letter was more effective in promoting apoptotic cell death with apparent hallmarks of apoptosis especially for A549 cells.

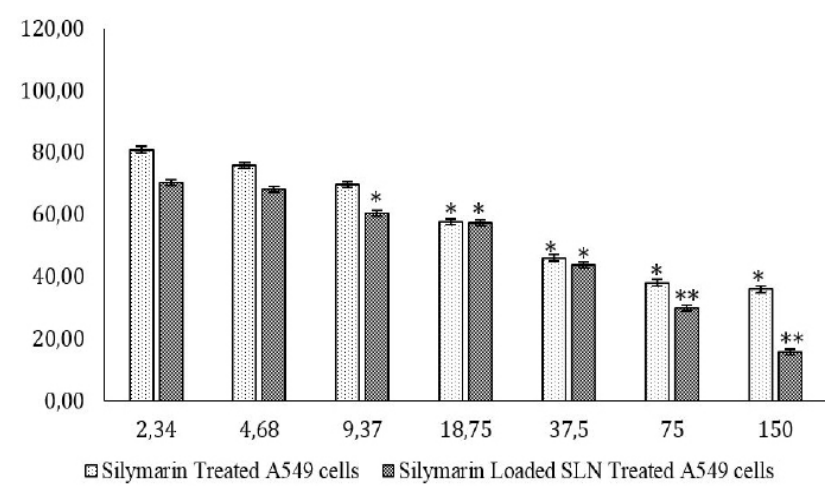

Fig. 2. Viability percentages of silymarin and silymarinloaded SLN treated A549 cells for $24 \mathrm{~h}$. $\mathrm{IC}_{50}$ value was detected to be $33 \mu \mathrm{M}$ (for silymarin) and $25 \mu \mathrm{M}$ (for silymarin-loaded SLN). ${ }^{*} \mathrm{p}<0.05, * * \mathrm{p}<0.01$

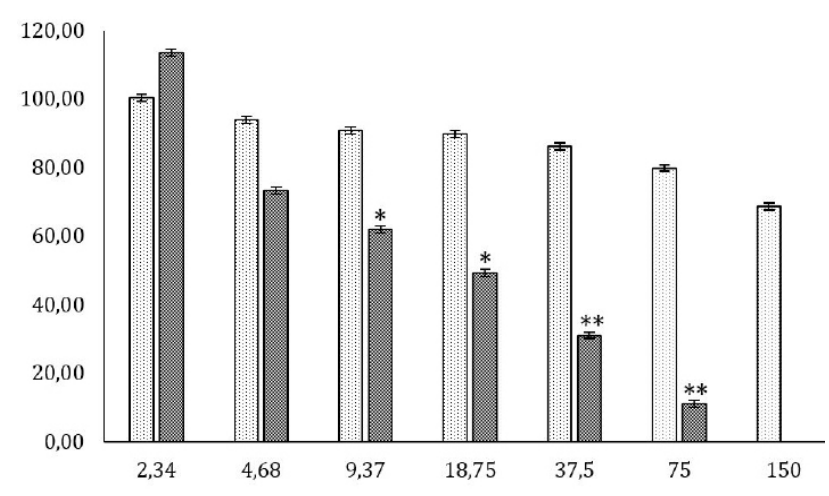

๑ Silymarin Treated MCF-7 cells — Silymarin Loaded SLN Treated MCF-7 cells

Fig. 3. Viability percentages of silymarin and silymarinloaded SLN treated MCF-7 cells for $24 \mathrm{~h}$. $\mathrm{IC}_{50}$ value for silymarin treated MCF-7 cells was not detected in this concentration range of the agent for $24 \mathrm{~h}$. $\mathrm{IC}_{50}$ value for silymarin-loaded SLN treated MCF-7 cells was detected to be $18 \mu \mathrm{M}$ for $24 \mathrm{~h} .{ }^{*} \mathrm{p}<0.05,{ }^{*} \mathrm{p}<0.01$

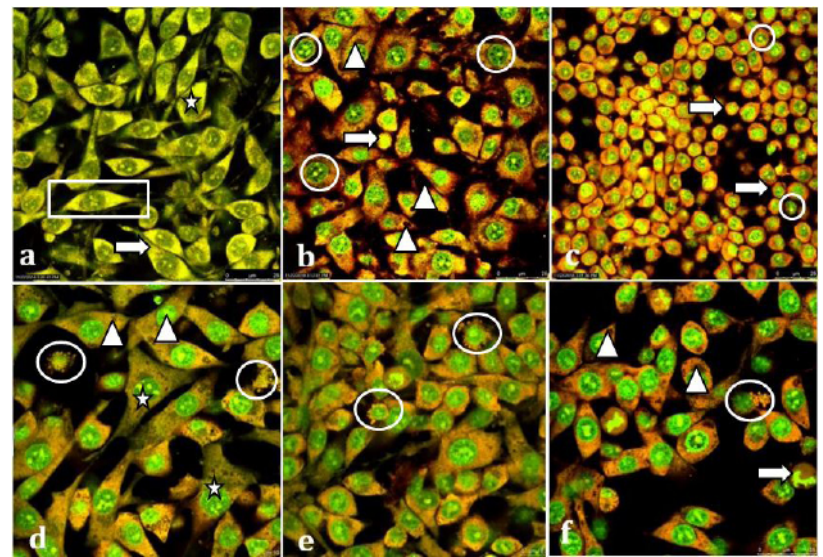

Fig. 4. Untreated A549 cells (a), astersk-nucleus, arrowcytoskeleton, rectangle-fusiform cell shape. A549 cells treated with $\mathrm{IC}_{50}$ value of silymarin (b and c), arrowshrunken (circular shaped) cells, arrow head-holes on cytoskeleton, circle-nuclei with condensed chromatin and A549 cells treated with $\mathrm{IC}_{50}$ value of silymarin-loaded SLN (d, e and f) for $24 \mathrm{~h}$; circle-cells with fragmented cytoskeleton, arrow-pyknotic nucleus, asterisk-chromatin condensation, arrow head-holes on cytoskeleton.

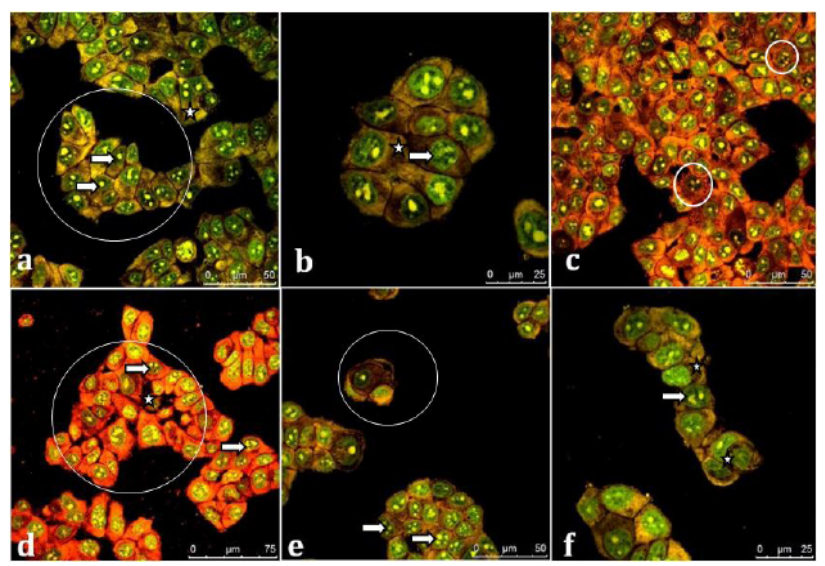

Fig. 5. Untreated MCF-7 cells (a and b); asteriskcytoskeleton, arrow-nuclei, circle-typical cell cluster. MCF-7 cells treated with $\mathrm{IC}_{50}$ value of silymarin (c and d); arrow-condensed chromatin, asterisk-condensed cell, circle-lacerated cell cluster, small circle-fragmented nuclei and MCF-7 cells treated with $\mathrm{IC}_{50}$ value of silymarin-loaded SLN (e and f) for $24 \mathrm{~h}$; circle-shrunken cell cluster with decreased number of cells, arrow- excessive chromatin condensation, asterisk-highly perforated cytoskeleton.

Cytotoxicity assays involve detection of cell death mode. The ultrastructural and physiological changes that occur during the death of cells can be visualized by transmission electron microscopic imaging that remains "gold standard" in this kind of examining. Some of these 
changes that can be taken into consideration for apoptotic cell death are reported as degradation of specific cellular proteins, condensation and fragmentation of nuclear chromatin as well as loss of membrane integrity of cellular membrane and organelles such as mitochondria (Kerr et al., 1972). In our study, in silymarin treated A549 and MCF-7 cells (Fig. 7), swollen mitochondria, lacerated cisterna of endoplasmic reticulum, disintegration of cytoskeleton with holes on it, granular cytoplasm, condensed chromatin (Fig. 6), secondary lysosomes, loss of cristae, granulated cytoplasm, cleavage of nuclear membrane (pyknotic nucleus) were detected TEM findings, respectively. However, in A549 and MCF-7 cells exposed to sylimarin-loaded SLNs for the same incubation time pyknotic nucleus, disintegrated mitochondrion with excessive loss of cristae, highly swollen cisterna of endoplasmic reticulum fragmentation of nucleus (Fig. 6), and chromatin condensation, disintegrated cell membrane and fragmented cytoskeleton containing huge holes (Fig. 7) were determined ultrastructural changes. From our TEM results it can be seen that SLNs treatment increased apoptosis when compared to silymarin alone application. Additionally, extensive cytoplasmic vacuolization was seen both in lung and breast cancer cells treated with SLNs implying an incompleted autophagy.

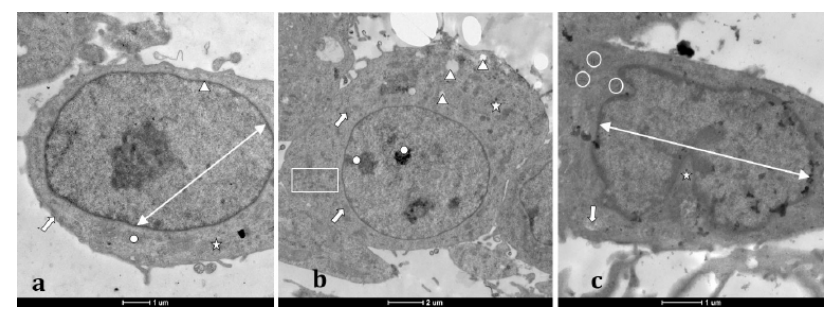

Fig. 6. Untreated A549 cell (a), arrow-cell membrane, twoheaded arrow-nucleus, circle-mitochondrion with compact cristae, asterisk-cytoskeleton. A549 cell treated with $\mathrm{IC}_{50}$ value of silymarin (b), Arrow-swollen mitochondria, rectangle-lacerated cisterna of endoplasmic reticulum, arrow head- holes on cytoskeletal disintegration, asteriskgranular cytoplasm, circle-condensed chromatin. A549 cell exposed to $\mathrm{IC}_{50}$ value of silymarin-loaded SLN for 24 $\mathrm{h}(\mathrm{c})$, two-headed arrow-pyknotic nucleus with extremely condensed chromatin, arrow-disintegrated mitochondrion with excessive loss of cristae, circle-highly swollen cisterna of endoplasmic reticulum and asterisk-fragmentation of nucleus.

In a study by Deep et al. (2007) silymarin was examined for its antiproliferative and anticancer activities in human breast, skin, prostate, cervical, and bladder cancers. They reported that silymarin triggers apoptotic cell death in colonic mucosa by reducing beta-glucuronidase activity and PGE2 level. Externalization of phosphatidylserine to the cell surface implies membrane disintegration and has been clear hallmark of early apoptosis (Kerr et al., 1972). These changes are determined by annexin-V staining in this study. Our results showed that silymarin caused total apoptotic cell death about $26 \%$ in A549 cells while about $36 \%$ of silymarin-loaded SLN treated A549 cells underwent apoptosis (Fig. 8). The percentage of total apoptotic cells in MCF-7 cells exposed to silymarin (Fig. 9) was found as $23 \%$. This percentage was detected to be about 34\% in silymarin-loaded SLN treated MCF-7 cells. These results refer to apparent high levels of apoptotic cell death derived from SLNs application. Similarly, in a study by Deep et al. (2007) silymarin and its nano formulations were reported to trigger apoptosis by arresting the cell cycle in G1 phase in LNCap and 22Rv1 cell lines (Deep et al., 2007).

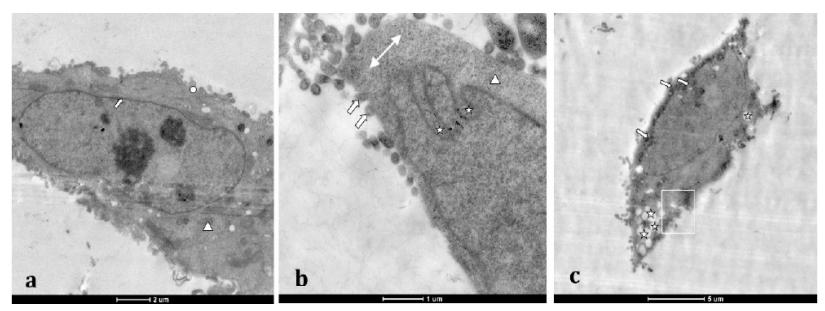

Fig. 7. Untreated MCF-7 cell (a), arrow-nuclear membrane, circle-cell membrane, arrow head-compact mitochondrion. MCF-7 cell treated with $\mathrm{IC}_{50}$ value of silymarin (b), Arrow-secondary lysosomes, Arrow headloss of cristae, two headed arrow-granulated cytoplasm, asterisk-cleavage of nuclear membrane (formation of pyknotic nucleus). Silymarin-loaded SLN treated MCF-7 cell (c), arrow-extreme chromatin condensation, rectangledisintegrated cell membrane and asterisk-fragmentation of the cytoskeleton (huge holes on cytoskeleton).

Recently, promoting apoptotic cell death by using natural agents in cancer treatment has been main research topic (Ghobrial et al., 2005). In this study, the investigated agents A549 and MCF-7 agents have triggered apoptosis but that of silymarin-loaded solid lipid particles was detected significantly higher than the silymarin alone. Silymarin and its newly synthesized Nano formulation showed cytotoxic, antiproliferative and apoptosis promoting activities on breast and lung cancer cells. Moreover, SLNs caused cytoplasmic vacuolization that imply an incompleted autophagy. Taken all together, these results are good fundamentals for understanding the anticancer efficacy of silymarin and silymarin-loaded solid lipid nano formulation to conquer the limitations of silymarin in anticancer research. Furthermore, the number of researches for preparing solid lipid nanoparticles as 
to use them as successful drug carriers with improved bioavailability have been in rise, recently. Thus, our results indicate the usage of drug delivery systems of colloidal characters to improve the pharmaceutical properties. It seems that sylimarin-loaded SLNs exhibit a quite good efficacy for treatment of lung and breast cancers offering strong theranostic potency.
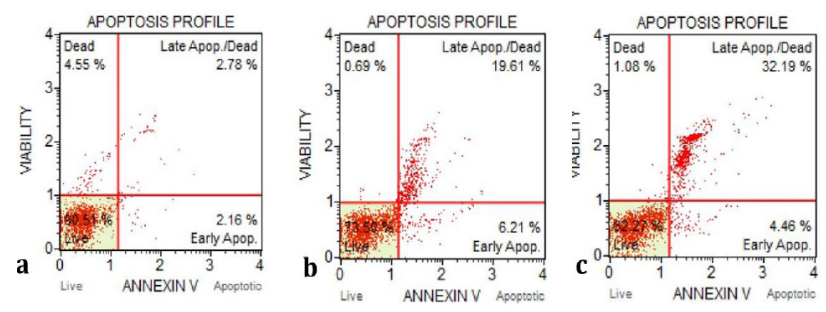

Fig. 8. Apoptosis profiles of untreated A549 cells (a) and A549 cells treated with $\mathrm{IC}_{50}$ values of silymarin (b) and silymarin-loaded SLN for $24 \mathrm{~h}$ (c). Apoptosis profiles of A549 control cells (a) showed $90.51 \%$ live, $2.16 \%$ early and $2.78 \%$ late apoptotic cells. In this group of cells percentage of dead cells was detected to be $4.55 \%$. A549 cells treated with $\mathrm{IC}_{50}$ concentration of silymarin (b) percentage of live cells was detected 73.50. Early apoptotic and late apoptotic cells of this group were $6.21 \%$ and $19.61 \%$, respectively. A negligible percentage $(0.69 \%)$ of the same group of A549 cells were dead. Total percentage of cells that underwent apoptosis was 36.75 of that $32.19 \%$ were in late apoptosis in silymarin-loaded SLN treated A549 cells (c). The percentage of live cells in the same group was detected to 62.27 for $24 \mathrm{~h}$.

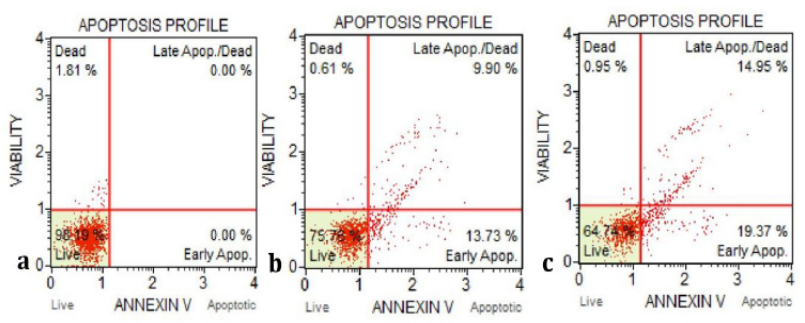

Fig. 9. Apoptosis profiles of untreated MCF-7 cells (a) and MCF-7 cells treated with $\mathrm{IC}_{50}$ values of silymarin (b) and silymarin-loaded SLN (c) for $24 \mathrm{~h}$. On the apoptosis profiles of untreated MCF-7 cells (a) were detected $98.19 \%$ live and $1.81 \%$ dead cells. In silymarin treated MCF-7 cells (b) the percentage of live cells was 75.76 and $13.73 \%$ and $9.90 \%$ of these cells were in early and late apoptotic stage, respectively. $0.61 \%$ of dead cells were detected in this group of cells. Early apoptotic and late apoptotic cells of this group were $6.21 \%$ and $19.61 \%$, respectively. Live cells percentage in silymarin-loaded SLN treated MCF-7 cells (c) was detected as $64.74 \%$. Early apoptotic and late apoptotic cell percentages were 19.37 and 14.95 for this cell group.

\section{CONCLUSIONS}

As a conclusion, our findings suggest that both silymarin and silymarin-loaded SLN formulation have been cytotoxic under lung and breast cancer cells in dose dependent manner, letter being more effective. Due to the apoptosis initiating efficacy and growth inhibition potency of silymarin-loaded SLNs at lower doses than silymarin alone both in two cell lines SLNs was considered as a solution for eliminating the limitations of silymarin. After the required deeper sequential investigations to figure out clearly its total molecular mechanism in a whole organism, silymarin-loaded SLNs might be a strong candidate for a sophisticate chemotherapeutic agent offering potentials for theranostic cancer treatment.

Statement of conflicts of Interest

The authors have declared no conflict of interest.

\section{REFERENCES}

Agarwal, R., Agarwal, C., Ichikawa, H., Singh, R.P. and Aggarwal, B.B., 2006. Anticancer potential of silymarin: From bench to bed side. Anticancer Res., 26: 4457-4498.

Al-Haj, N. and Rasedee, A., 2009. Solid lipid nanoparticles preparation and characterization. Sci. Alert, 1: 90-93. https://doi.org/10.3923/ ijp.2009.90.93

Aljuffali, I.A., Fang, C.L., Chen, C.H. and Fang, J.Y., 2016. Nanomedicine as a strategy for natural compound delivery to prevent and treat cancers. Curr. Pharm. Design., 22: 4219-4231. https://doi. org/10.2174/1381612822666160620072539

Almeida, H., Lobão, P., Frigerio, C., Fonseca, J., Silva, R. and Palmeira-de-Oliveira, A., 2015. New thermoresponsive eyedrop formulation containing ibuprofen loaded-nanostructured lipid carriers (NLC): Development, characterization and biocompatibility studies. Curr. Drug. Deliv., 22: 336-349.

Chih-Hung, L., Chun-Han, C., Zih-Chan, L. and JiaYou, F., 2017. Recent advances in oral delivery of drugs and bioactive natural products using solid lipid nanoparticles as the carriers. J. Fd. Drug. Anal., 25: 1-16.

Deep, G.N.H., Oberlies, D.J., Kroll, R., and Agarwal, 2007. Isosilybin B and isosilybin A inhibit growth, induce G1 arrest and cause apoptosis in human prostate cancer $\mathrm{LNCaP}$ and $22 \mathrm{Rv} 1$ cells. Carcinogenesis, 28: 1533-1542. https://doi. org/10.1093/carcin/bgm069 
Eminzade, S., Uraz, F. and Izzettin, F.V., 2008. Silymarin protects liver against toxic effects of antituberculosis drugs in experimental animals. Nutr. Metab., pp. 5-18. https://doi.org/10.1186/17437075-5-18

Geszke-Moritz, M. and Moritz, M., 2016. Solid lipid nanoparticles as attractive drug vehicles: Composition, properties and therapeutic strategies. Mater. Sci. Eng. C Mater. Biol. Appl., 68: 982-994. https://doi.org/10.1016/j.msec.2016.05.119

Gezgin, D., Kabadere, S. and Kuş, G., 2013. Comparing the role of silymarin on survival rate of rat glioma with primary mixed rat glia cells in vitro. Fresen. Environ. Bull., 22: 443-448.

Gharagozloo, M., Karimi, M. and Amirghofran, Z., 2013. Immunomodulatory effects of silymarin in patients with $\beta$-thalassemia major. Int. Immunopharmacol., 16: 243-247. https://doi. org/10.1016/j.intimp.2013.04.016

Ghobrial, I.M., Witzig, T.E. and Adjei, A.A., 2005. Targeting apoptosis pathways in cancer therapy. CA Cancer J. Clin., 55: 178-194. https://doi. org/10.3322/canjclin.55.3.178

Harde, H., Das, M. and Jain, S., 2011. Solid lipid nanoparticles: An oral bioavailability enhancer vehicle. Expert. Opin. Drug Deliv., 8: 1407-1424. https://doi.org/10.1517/17425247.2011.604311

Iriti,M. andFaoro,F.,2009. Bioactivity of grapechemicals for human health. Nat. Prod. Commun., 4: 611-634. https://doi.org/10.1177/1934578X0900400502

Jaafar-Maalej, C., Andrieu, V., Elaissari, A. and Fessi, H., 2011. Beclomethasone- loaded lipidic nanocarriers for pulmonary drug delivery: preparation, characterization and in vitro drug release. J. Nanosci. Nanotechnol., 11: 1841-1851. https://doi.org/10.1166/jnn.2011.3119

Jabir, NR., Tabrez, S., Ashraf, GM., Shakil, S., Damanhouri, GA. and Kamal, MA., 2012. Nanotechnology-based approaches in anticancer research. Int. J. Nanomed., 7: 4391-4408. https:// doi.org/10.2147/IJN.S33838

Karimi, G., Vahabzadeh, M., Lari, P., Rashedinia, M. and Moshiri, M., 2011. Silymarin, a promising pharmacological agent for treatment of diseases. Iran. J. Basic med. Sci., 14: 308-317.

Kerr, J.F.R., Wyllie, A.H. and Currie, A.R., 1972. Apoptosis: A basic biological phenomenon with wide-ranging implications in tissue kinetics. $\mathrm{Br}$. J. Cancer, 26: 239-257. https://doi.org/10.1038/ bjc. 1972.33

Li, S.D. and Huang, L., 2008. Pharmacokinetics and biodistribution of nanoparticles. Mol. Pharm., 5:
496-504. https://doi.org/10.1021/mp800049w

Liu, R.H., 2004. Potential synergy of phytochemicals in cancer prevention: mechanism of action. $J$. Nutr., 134: 3479-3485. https://doi.org/10.1093/ jn/134.12.3479S

Malewicz, B., Wang, Z., Jiang, C., Guo, J., Cleary, M.P. and Grande, J.P., 2006. Enhancement of mammary carcinogenesis in two rodent models by silymarin dietary supplements. Carcinogenesis, 27: 17391747. https://doi.org/10.1093/carcin/bg1032

Mayer, K.E., Mayer, R.P. and Lee, S.S., 2005. Silymarin treatment of viral hepatitis: a systematic review. $J$. Viral Hepat., 12: 559-567. https://doi.org/10.1111/ j.1365-2893.2005.00636.x

Montgomery, A., Adeyeni, T., San, K., Heuertz, R.M. and Ezekiel, R.U., 2016. Curcumin sensitizes silymarin to exert synergistic anticancer activity in colon cancer cells. J. Cancer, 7: 1250-1257. https:// doi.org/10.7150/jca. 15690

Muller, R.H., Dingler, A. and Schneppe, T., 2000. Large scale production of solid lipid nanoparticles $\left(\mathrm{SLN}^{\mathrm{TM}}\right)$ and nanosuspensions (Disso Cubes R). In: Handbook of pharmaceutical controlled release technology (ed. D. Wise). $2^{\text {nd }}$ ed. Marcel Dekker, New York. pp. 359-376. www.sigmaaldrich.com

Numanoğlu, U. and Tarımc1, N., 2006. Characterization of solid lipid nanoparticles (SLNTM) and their pharmaceutical and cosmetic applications. Ankara J. Fac. Pharm., 35: 211-235. https://doi. org/10.1501/Eczfak_0000000061

Obradovic, A., Zizic, J., Trisovic, N., Bozic, J., Uscumlic, G., Bozic, B., 2013. Evaluation of antioxidative effects of twelve 3-substituted-5,5diphenylhydantoins on human colon cancer cell line HCT-116. Turk. J. Biol., 37: 741-747. https:// doi.org/10.3906/biy-1302-15

Özkan, A. and Erdoğan, A., 2013. Membrane and DNA damaging/protective effects of eugenol, eucalyptol, terpinen-4-ol, and camphor at various concentrations on parental and drug resistant H1299 cells. Turk. J. Biol., 37: 405-413. https://doi. org/10.3906/biy-1208-4

Polyak, S.J., Morishima, C., Lohmann, V., Pal, S., Lee, DY., Liu, Y., Graf, T.N. and Oberlies, N.H., 2010. Identification of hepatoprotective flavonolignans from silymarin. Proc. natl. Acad. Sci. U.S.A., 107: 5995-5999. https://doi.org/10.1073/ pnas.0914009107

Prasad, N.R., Muthusamy, G., Shanmugam, M. and Ambudkar, SV., 2016. South Asian medicinal compounds as modulators of resistance to chemotherapy and radiotherapy. Cancers (Basel), 
pp. 8-32. https://doi.org/10.3390/cancers8030032

Provinciali, M., Papalini, F., Orlando, F., Pierpaoli, S., Morazzoni, P.D.A. and Riva, A., 2007. Effect of the silybin-phosphatidylcholine complex (IdB 1016) on the development of mammary tumors in HER-2/ neu transgenic mice. Cancer Res., 67: 2022-2029. https://doi.org/10.1158/0008-5472.CAN-06-2601

Seyfoddin, A., Sherwin, T., Patel, D.V., McGhee, C.N., Rupental, I.D. and Taylor, J.A., 2015. Ex vivo and In vivo evaluation of chitosan coated nanostructured lipid carriers for ocular delivery of acyclovir. Curr.
Drug Deliv., 13: 923-934. https://doi.org/10.2174/ 1567201813666151116142752

Torre, L.A., Bray, F., Siegel, R.L., Ferlay, J., LortetTieulent, J. and Ahmedin, J., 2015. Global cancer statistics. CA Cancer J. Clin., 65: 87-108. https:// doi.org/10.3322/caac. 21262

Venkateswarlu, V. and Manjunath, K., 2004. Preparation, characterization and in vitro release kinetics of clozapine solid lipid nanoparticles. $J$. Contr. Rel., 95: 627-638. https://doi.org/10.1016/j. jconrel.2004.01.005 